\title{
Real-time three-dimensional video image composition by depth information
}

\author{
Masahiro Kawakita, ${ }^{1 \mathrm{a})}$ Keigo Iizuka, ${ }^{2 \mathrm{~b})}$ Tahito Aida, ${ }^{3}$ \\ Taiichirou Kurita, ${ }^{1}$ and Hiroshi Kikuchi ${ }^{1}$ \\ ${ }^{1}$ Science and Technical Research Laboratories, Japan Broadcasting Corporation. \\ 1-10-11 Kinuta, Setagaya-ku, Tokyo 157-8510, Japan \\ ${ }^{2}$ Depertment of Electrical \& Computer Engineering, University of Toronto. \\ Ontario, M5S 1A4, Canada \\ ${ }^{3}$ Department of Electrical Engineering, Graduate School of Engineering, Osaka \\ City University, 3-3-138 Sugimoto, Sumiyoshi-ku, Osaka 558-8585, Japan \\ a) kawakita.m-ga@nhk.or.jp \\ b)keigo.iizuka@utoronto.ca \\ b) http://www.keigo-iizuka.com
}

Abstract: We have developed a system of compositing threedimensional (3D) video images based on the depth information of the objects. The system consists of the Axi-Vision Camera that can measure object distance in real time and an arithmetic image processor that can synthesize video images according to the depth information. The paper demonstrates how to three-dimensionally synthesize such a scene as an array of computer-generated characters moving around a standing person in real time. The feasibility of using such a signal processor to create realistic TV programs in a broadcasting station has been studied.

Keywords: three-dimensional image, image composition, depth image

Classification: Science \& engineering for electronics

\section{References}

[1] S. Shimoda, M. Hayashi, and Y. Kanatsugu, "New chroma-key imaging technique with Hi-Vision background," IEEE Trans. Broadcast., vol. 35, no. 4, pp. 357-361, Dec. 1989.

[2] C. Swain and T. Chen, "Defocus-based image segmentation," Proc. IEEE Int. Conf. On Acoustics, Speech, and Signal Processing, Detroit, pp. 24032406, May 1995.

[3] T. Kanade, A. Yoshida, K. Oda, H. Kano, and M. Tanaka, "A stereo machine for video-rate dense depth mapping and its new applications," Proc. of the 15th Computer Vision and Pattern Recognition Conference (CVPR), San Francisco, USA, pp. 196-202, June 1996.

[4] M. Kawakita, K. Iizuka, T. Aida, H. Kikuchi, H. Fujikake, J. Yonai, and K. Takizawa, "Axi-Vision Camera (real-time distance-mapping camera)," Appl. Opt., vol. 39, no. 22, pp. 3931-3939, Aug. 2000. 
[5] W. Woo, N. Kim, and Y. Iwadate, "Object segmentation for Z-keying using stereo images," Proc. of World Computer Congress - International Conference on Signal Processing (WCC-ICSP) 2000, Beijing, pp. 12491254, Aug. 2000.

[6] M. Kawakita, K. Iizuka, H. Nakamura, I. Mizuno, T. Kurita, T. Aida, Y. Yamanouchi, H. Mitsumine, T. Fukaya, H. Kikuchi, and F. Sato, "Highdefinition real-time depth-mapping TV camera: HDTV Axi-Vision Camera," Opt. Express, vol. 12, no. 12, pp. 2781-2794, June 2004, http://www.opticsexpress.org/abstract.cfm?URI=OPEX-12-12-2781.

[7] H. Kikuchi and Y. Yamanouchi, "BS Hi-Vision Utukusiki Nihon Hyaku no Fuukei Special Production Report -New production method in a new generation virtual studio-," (in Japanese) Hohsoh gijutsu, vol. 4, pp. 167174, April 2003.

\section{Introduction}

Recently, the demand for a more realistic as well as a higher-quality composite video image has increased with the progress of digital technologies in the movie and broadcasting industries. Chroma-keying is a well established method that segments the image of particular objects from the video image using color information of a designated color screen [1]. This method, however, requires a large blue or green back-screen. With this method, actors are not allowed to wear clothes of the same color as that of the screen. Depthkeying is a newer method, which is starting to be used for segmenting objects located at a particular distance from the camera [2-5] to eliminate the need for a designated color screen.

We have developed the Axi-Vision Camera $[4,6]$, a real-time depthmapping camera. Combing this camera with a high speed arithmetic image processor, the creation of realistic 3D images has been made possible. In the process of combining more than one image into one, the processor uses the depth information to determine whether a particular segment of the image is to be extinguished or distinguished. This paper explains how the composite is formed using the example of a live person with an array of moving computer graphic characters.

\section{System}

The principle of distance measurement of the Axi-Vision Camera is based on the fact that the intensity of an image taken by an ultrafast shutter speed camera becomes dependent on the distance between the camera and the object as the speed of modulation of the illuminating light approaches the speed of light [4]. Figure 1 shows a block diagram of the system. A triangular wave signal and shutter triggering signal are provided from one main function generator. The control signals for switch 1 and 2 are the vertical synchronizing video signals of the color camera. Switch 1 separates the ascending and descending periods of the triangular signal for modulating the output light of the LED illuminator. The shutter triggering signal opens 


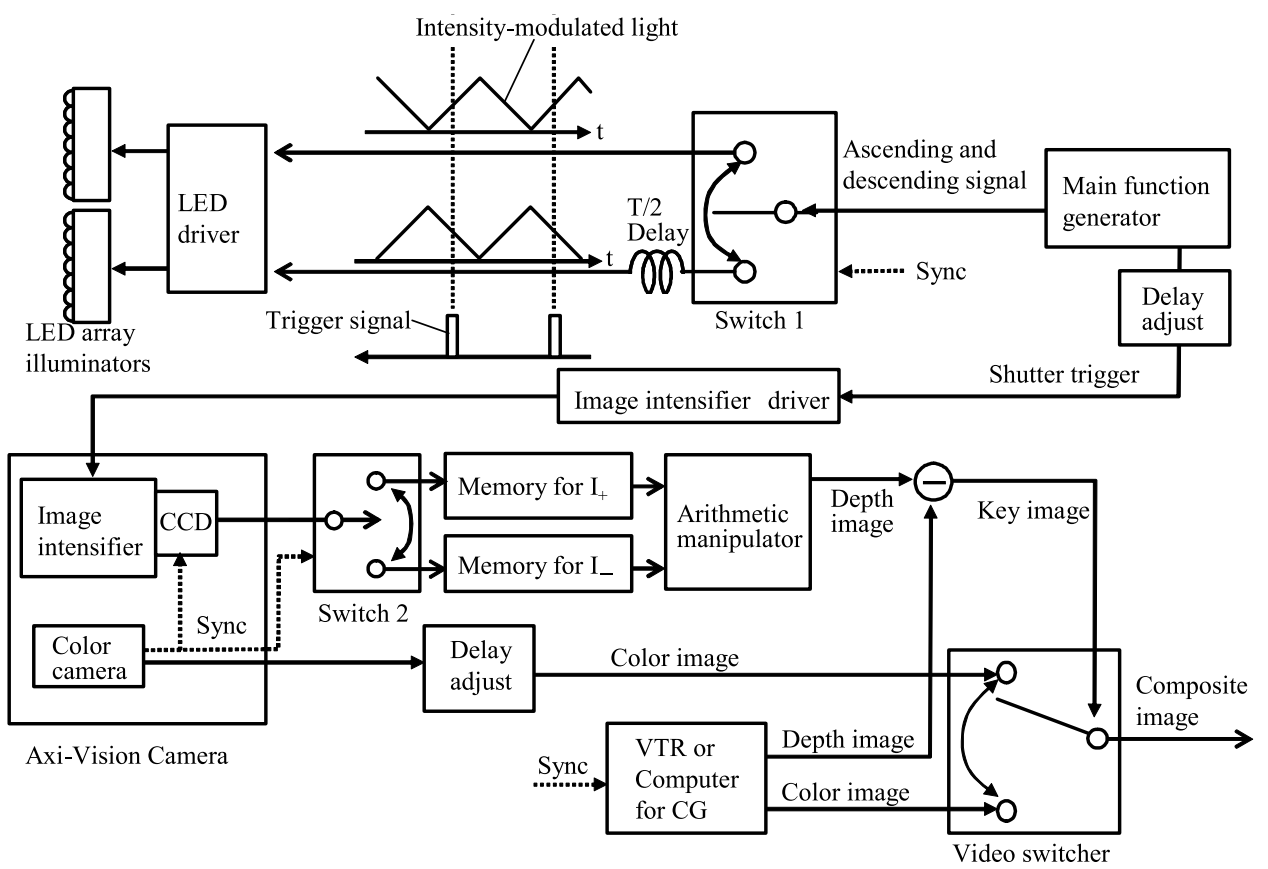

Fig. 1. Block diagram of the system.

the shutter of the image intensifier tube in front of the CCD camera at an appropriate time. Switch 2 sorts out the output $\mathrm{I}_{+}(\mathrm{x}, \mathrm{y})$ of the CCD camera taken with ascending illumination from the output $\mathrm{I}_{-}(\mathrm{x}, \mathrm{y})$ taken with descending illumination. These two outputs are accumulated in the respective frame memories.

The time sequence of the accumulation in the frame memories is detailed in Fig. 2. To detect a depth image of the object in real time, $I_{+}$and $I_{-}$are taken in every video frame (a minimum of 1 /60 seconds) alternately. The depth image is calculated using $\mathrm{I}_{+}$and $\mathrm{I}_{-}$fore and aft frames. Thus, the depth image can be updated at the video frame rate and the system can detect depth images of moving objects in real time. The depth is calculated by

$$
d(x, y)=\frac{1}{2}\left[1-k \frac{I_{-}(x, y)-I_{+}(x, y)}{I_{-}(x, y)+I_{+}(x, y)}\right]
$$

where $k$ is a constant [4].

It should be pointed out that in connection with selecting the shutter time of the image intensifier tube there are conflicting requirements of shorter time for higher resolution and longer time for a better signal-to-noise ratio of the output. As a solution to this problem, the shutter is repeatedly opened at about 750,000 times during one frame of $1 / 60 \mathrm{sec}$ and the results are accumulated. This accumulation improved the SNR by a factor of 866 .

The output depth video image has 10 bit data with $1280 \times 720$ pixels at $29.97 \mathrm{~Hz}$, or $853 \times 480$ pixels at $59.94 \mathrm{~Hz}$. The SNR of the output depth image of the HDTV signal is $44.2 \mathrm{~dB}$ at an object distance of $2 \mathrm{~m}$. The signal processor for calculation of the depth image is executed by FPGA (Field Programmable Gate Arrays). The contrast of a depth image is adjusted by controlling the coefficient $k$ of Eq. (1). The size of the depth image is adjusted 


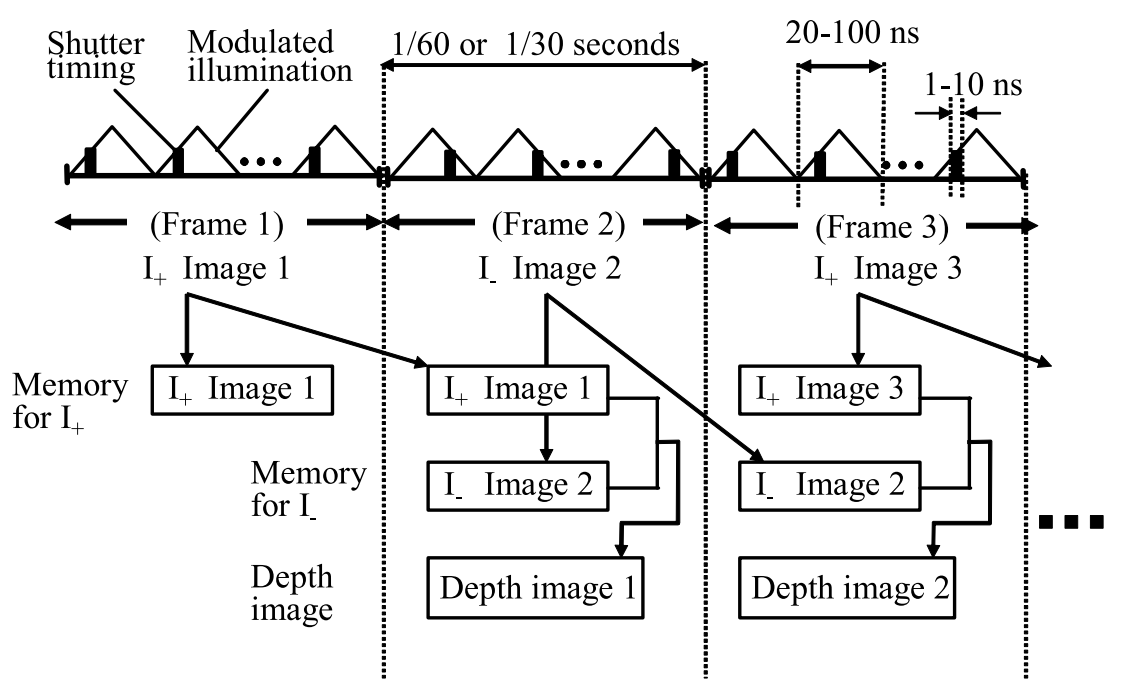

Fig. 2. Time chart of image processing for calculating the depth image.

to that of the color image to the accuracy of one pixel for high precision image composition.

\section{Image composition experiment}

Figure 3 shows the procedure for the 3D video image composition. The image composition can be realized by comparing the depth information of the real image with that of the computer-generated (CG) image.

The relative position in depth between the real object and the CG characters is calculated by taking the difference between the depth $\mathrm{D}_{1}$ of the real object (Fig. 3 (a)) and $\mathrm{D}_{2}$ of the CG (Fig. $3(\mathrm{c})$ ) for each pixel. The difference

$$
\Delta D(x, y)=D_{2}(x, y)-D_{1}(x, y)
$$

provides the relative distance between the real object and the CG characters. If $\Delta D>0$, the distance of the pixel of the CG image is further than that of the real object, and if conversely $\Delta D<0$, the distance of the pixel of the real object is further than that of the CG image. Figure $3(\mathrm{e})$ shows the pattern of $\Delta D$ where an offset value was added so that all $\Delta D$ values become positive.

The key image was generated by quantizing the density of Fig. 3 (e) into two levels of black and white as shown in Fig. 3 (f). The key image is used to determine which pixel of the real image or CG images is extracted at a specific location. In the region of the key image in black, the CG image is extracted and in the region in white, the real image is extracted.

In the composite image (Fig. $3(\mathrm{~g})$ ), CG characters that are farther to the camera than the person are hidden behind the person, and the CG characters that are nearer the camera than the person are displayed in front of the person.

Image synthesis by the chroma-keying method merely superimposes the extracted image over the paired image, regardless of their distance relation- 


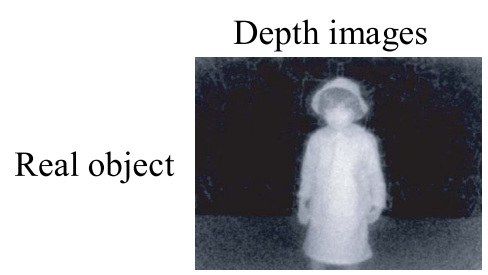

(a)

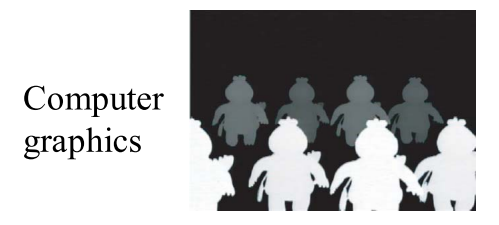

(c)

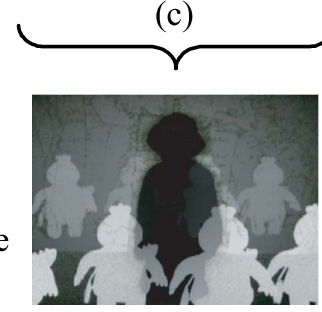

(e)
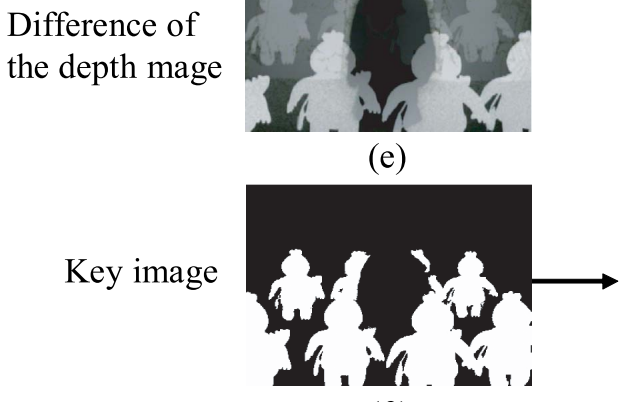

(f)

\section{Color images}

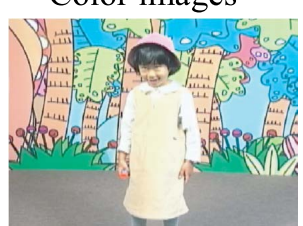

(b)

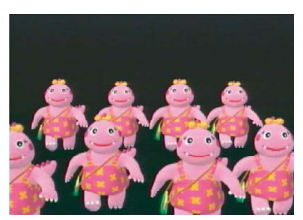

(d)

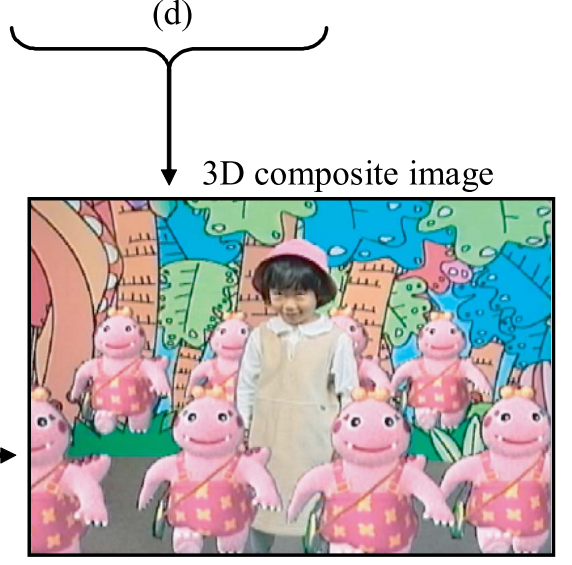

(g)

Fig. 3. A real image and CG are combined into a 3D composite image (a) Depth image and (b) color image of the real object, (c) depth image and (d) color image of the CG, (e) difference between the depth images (f) key image, and (g) 3D composite image displayed in the NHK Learning Fairs 2001 and 2003. Movie file is attached.

ship. On the other hand, 3D image composition by the depth-keying method extracts specific pixels according to the depth information of the object. Unlike the conventional chroma-keying, it is possible to create such virtual scenery that appears as if the person and the CG characters exist in the same space in real-time even when the objects are in motion.

This 3D image composition method has been applied to an actual HDTV program production $[6,7]$. It demonstrated that the 3D image composition method can produce realistic virtual studios for TV broadcasting stations.

\section{Conclusion}

We developed a real-time 3D image composition system that combines a real image with CG image using depth information. The application of the system is not limited to realizing effective production of a virtual studio in TV programming. It has the potential to be used in a much wider range of fields, such as 3D TV, digital cinema, the game industry, and medical treatment. 


\section{Acknowledgments}

The authors would like to thank Visual Design Corporation for their technical support in developing the image processing system. We also thank Kenji Kikue and all the staff at the NHK Broadcasting Corporation engaged in the program production with this system. Mary Jean Giliberto's proofreading of the manuscript is very much appreciated. 